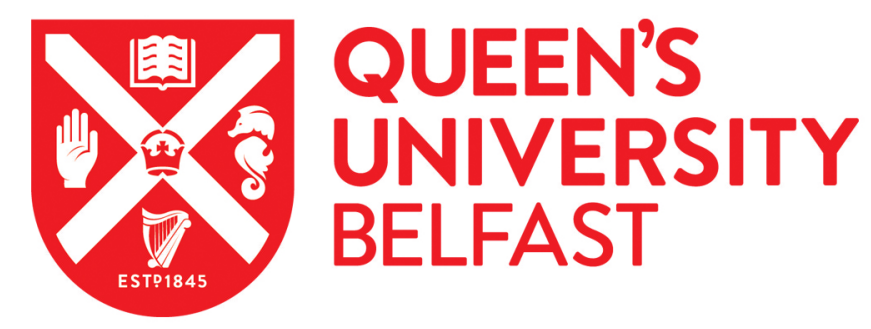

\title{
Is the flow tube reactor with NO2 titration a reliable absolute source for atomic hydrogen?
}

Niemi, K., Mosbach, T., \& Döbele, H. F. (2003). Is the flow tube reactor with NO2 titration a reliable absolute source for atomic hydrogen? Chemical Physics Letters, 367(5-6), 549-555. https://doi.org/10.1016/S00092614(02)01742-6

Published in:

Chemical Physics Letters

Queen's University Belfast - Research Portal:

Link to publication record in Queen's University Belfast Research Portal

\section{General rights}

Copyright for the publications made accessible via the Queen's University Belfast Research Portal is retained by the author(s) and / or other copyright owners and it is a condition of accessing these publications that users recognise and abide by the legal requirements associated with these rights.

Take down policy

The Research Portal is Queen's institutional repository that provides access to Queen's research output. Every effort has been made to ensure that content in the Research Portal does not infringe any person's rights, or applicable UK laws. If you discover content in the Research Portal that you believe breaches copyright or violates any law, please contact openaccess@qub.ac.uk. 


\title{
Is the flow tube reactor with $\mathrm{NO}_{2}$ titration a reliable absolute source for atomic hydrogen?
}

\author{
K. Niemi, T. Mosbach, H.F. Döbele * \\ Institut für Laser- und Plasmaphysik, Universität Essen, D-45117 Essen, Germany
}

Received 24 May 2002; in final form 24 May 2002

\begin{abstract}
The $\mathrm{H}+\mathrm{NO}_{2}$ titration scheme for the determination of atomic hydrogen densities within a microwave excited flow tube reactor has been investigated by laser-induced fluorescence spectroscopy in the vacuum UV. Absolute hydrogen densities are determined on the basis of calibration by Rayleigh scattering from argon. The measurement is performed at a gas mixture containing $0.5 \%$ of $\mathrm{D}_{2}$ added to the main gas $\mathrm{H}_{2}$. The ground state density of the hydrogen atoms generated in the flow tube reactor was inferred from the fluorescence radiation of the spectrally shifted optically thin D-Lyman- $\alpha$ transition.

(c) 2002 Published by Elsevier Science B.V.
\end{abstract}

\section{Introduction}

The availability of atomic reference sources with quantitatively known density is of considerable importance for the measurement of crosssections for excitation and ionization both by particle collisions and by interactions with photons. It is of particular importance for the calibration of two-photon laser-induced fluorescence spectroscopy. This experimental technique is applied to measure atomic ground state populations with space and time resolution in fusion plasmas, processing plasmas or flames. The atomic species is usually excited from the ground-state with the aid

\footnotetext{
${ }^{*}$ Corresponding author. Fax: +49-201-183-3029.

E-mail address: doebele@uni-essen.de (H.F. Döbele).

URL: http://www.ilp.physik.uni-essen.de/doebele.
}

of narrow-bandwidth laser radiation into a higher state. The subsequent fluorescence radiation allows to infer the original ground state population. Absolute densities are often determined on the basis of reference sources which are assumed to generate well-defined atomic densities. Flow tube systems with microwave discharges are widely used; the generated atomic density is determined on the basis of a titration process [1]. Complications by collisional quenching at elevated pressure will be covered only briefly in this contribution.

An alternative calibration method has come up recently which needs no reference source [2]. It is based on a two-photon laser-induced fluorescence measurement (TALIF) of a noble gas which has a two-photon resonance spectrally close to that of the investigated atomic species. The ratio of the two-photon absorption cross-sections has to be 
known in order to make this method applicable. Some important two-photon cross-section ratios have been determined for atomic hydrogen, nitrogen and oxygen [3] - the measurement was based, however, on flow tube reactors in which the atomic density had been determined by titration. This makes clear that the question of reliability of the titration in flow-tube reactors is of great importance for absolute atomic density measurements.

We report in this Letter on the first direct test of the widely used titration scheme $\mathrm{H}+\mathrm{NO}_{2}$. We generate in a conventional flow tube reactor atomic hydrogen/deuterium densities which we diagnose by single-photon laser-induced fluorescence spectroscopy on the Lyman- $\alpha$ transition. Absolute ground state densities are determined both by titration and by Rayleigh calibration with argon whereby the latter is considered to be quantitatively reliable so that it can serve as a standard for the titration technique. Tunable narrow-bandwidth coherent VUV radiation $(\lambda=121.5 \mathrm{~nm})$ is generated by stimulated antiStokes Raman scattering (SARS) from $\mathrm{LN}_{2}-$ cooled molecular hydrogen. The H-Lyman- $\alpha$ transition is optically thick for typical operational parameters of the flow tube reactor. We have solved this problem by adding a well-defined fractional amount of deuterium to the working gas. The spectrally shifted D-Lyman- $\alpha$ transition $\left(\Delta v=22.5 \mathrm{~cm}^{-1}\right)$ is optically thin and allows to perform the quantitative measurement. The basic assumption is that the atomic isotope ratio is identical to the molecular isotope ratio in view of the similarity of the generation and loss processes in the flow tube reactor.

\section{Experimental arrangement}

\subsection{LIF setup}

The laser system for VUV generation consisted of a XeCl excimer laser (Lambda Physik LPX301i, $\lambda=308 \mathrm{~nm}, E=500 \mathrm{~mJ}, \Delta t=20 \mathrm{~ns})$, by which a two-stage dye laser (Lambda Physik FL 301e) was pumped. The dye laser radiation (Coumarine 120, $\lambda=445.5 \mathrm{~nm}, E=30-60 \mathrm{~mJ}, \Delta t=15 \mathrm{~ns})$ was fre- quency doubled in a BBO crystal. The generated UV radiation $\left(E=4-8 \mathrm{~mJ}, \Delta v=0.2 \mathrm{~cm}^{-1}\right)$ was singled out by two dielectric mirrors from the fundamental wave and was focussed into a Raman cell $(R=+65$ $\mathrm{mm}$ ) where the required VUV radiation of wavelength $\lambda_{\mathrm{D}}=121.54 \mathrm{~nm}$ was generated by SARS [4]. Both the pump radiation and all Stokes and antiStokes products left the Raman cell. A concave grating (Milton Roy, $R=995.4 \mathrm{~mm}$, lines 1200/ $\mathrm{mm}$, blaze wavelength $120 \mathrm{~nm}, \mathrm{MgF}_{2}$-coating) provided the spectral separation of the various radiation fields. The ninth anti-Stokes component was singled out in our case. Tuning the dye laser provided then narrow-bandwidth tunable coherent VUV radiation suitable to excite the Lyman- $\alpha$ transitions (see Fig. 1).

The VUV radiation was guided under vacuum in order to prevent absorption. The imaging grating provided a slightly converging conical beam of radiation extending to the flow tube reactor. It passed a $1 \mathrm{~m} \mathrm{McPherson} \mathrm{monochromator}$ and was detected by a solar blind photomultiplier (Hamamatsu R1259) in the image plane of the instrument. Laser-induced fluorescence radiation was detected with a solar blind photomultiplier of the same type at $90^{\circ}$ to the incoming beam. Two plano-convex lenses $(d=40 \mathrm{~mm}, R=+75 \mathrm{~mm})$ made of high quality monocrystalline $\mathrm{MgF}_{2}$ provided the imaging of the scattering volume onto the detector. A narrow-bandwidth Lyman- $\alpha$ interference filter (Acton Research, central wavelength $121.3 \mathrm{~nm}$, peak transmission 9\%, FWHM $8.4 \mathrm{~nm}$ ) was placed in front of the second photomultiplier to suppress parasitic radiation. The re-

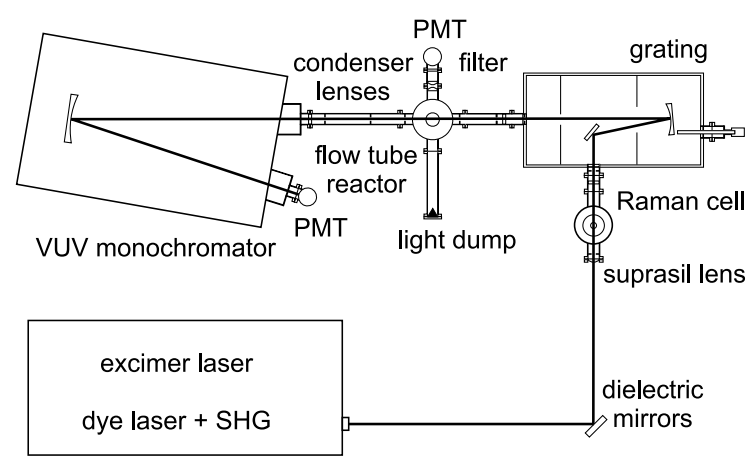

Fig. 1. Experimental setup. 
liable operation of the photomultipliers was checked with respect to linearity.

\subsection{Flow tube reactor}

Fig. 2 shows the flow tube reactor system with the microwave discharge. The composition of the gas mixture $\left(0.5 \% \mathrm{D}_{2}, 99.5 \% \mathrm{H}_{2}\right.$, mass spectroscopic certificate) was provided by Messer-Griesheim. The hydrogen was diluted in helium and was fed to the quartz tube (inner diameter $9 \mathrm{~mm}$ ) coaxial with the cylindrical resonator of the microwave discharge $(2.45 \mathrm{GHz})$. The central part of the flow tube reactor consisted of a Teflon tube (inner diameter $19 \mathrm{~mm}$ ) in which both the flow of the microwave discharge and the titration gas were mixed. The resulting flow remained laminar. Four holes in this flow tube provided access for the optical beams. The titration gas was added through a Teflon tube $6 \mathrm{~cm}$ above the observation

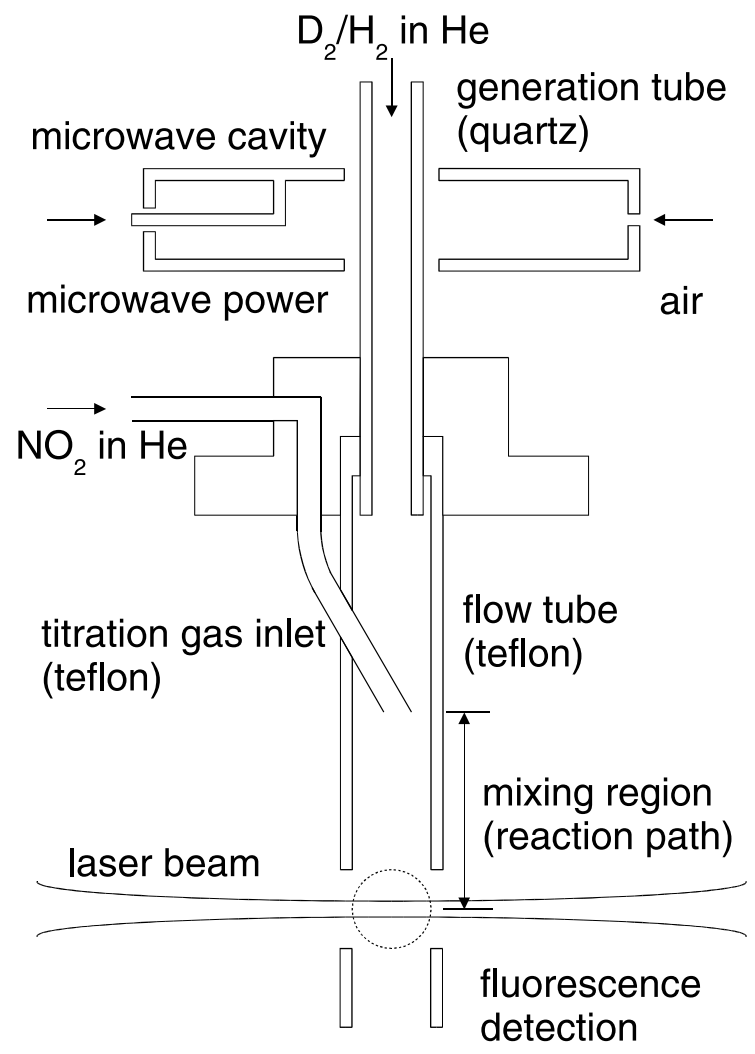

Fig. 2. Flow tube reactor. volume. The titration gas was a mixture of $\mathrm{NO}_{2}$ and helium; the $\mathrm{NO}_{2}$ fraction amount to $1.47 \%$ was determined with the aid of an FTIR spectrometer. The large fraction of the inert gas helium warranted an efficient and stable discharge even with variable fluxes of the titration gas. The wall material had been chosen to minimize recombination losses of atomic species. In addition, the quartz tube was treated with hydrofluoric acid. The fluxes of all gases were controlled by mass flow meters; the total pressure was monitored by a capacitive manometer independent of the type of gas. The gas purity was better than $99.7 \%$ for deuterium and $99.999 \%$ for hydrogen. The purity of helium was guaranteed by the supplier to be better than $99.99999 \%$, so that the flux of possible impurities in the working gas under typical operational conditions was always less than $1 \%$ of the $\mathrm{D}_{2}$ flux. The flow tube reactor was attached to a vacuum chamber; the total flow was controlled by a throttle valve.

\section{Measurements}

\subsection{D-Lyman- $\alpha$ fluorescence signal}

The number of detected fluorescence photons per laser pulse is given by

$\phi_{\mathrm{F}}\left(\lambda_{\mathrm{L}}\right)=T \Delta \Omega L K(\theta) \sigma^{\lambda} G\left(\lambda_{\mathrm{D}}-\lambda_{\mathrm{L}}\right) \phi_{\mathrm{L}} n$.

This formula applies for an optically thin medium without quenching and saturation. $\lambda_{\mathrm{D}}$ is the wavelength of the resonance, $\lambda_{\mathrm{L}}$ is the central wavelength of the exciting radiation, $T$ is the transmission of the detection optics, $\Delta \Omega$ is the solid angle of detection, $L$ is the length of the observed volume, $K(\theta)$ describes the fluorescence radiation characteristic, $\sigma^{\lambda}$ is the excitation cross-section integrated over the wavelength, $G\left(\lambda_{\mathrm{D}}-\lambda_{\mathrm{L}}\right)$ is the normalized profile of excitation $\left(\int G(\lambda) \mathrm{d} \lambda=1\right)$ which results from the convolution of the atomic transition line profile with the profile of the exciting radiation, $\phi_{\mathrm{L}}$ is the number of laser photons and $n$ is the ground state density of D-atoms in the volume of observation. The wavelength integrated excitation cross-section, summed over the $2 \mathrm{p}^{2} \mathrm{P}_{J}$ finestructure, is calculated according to 
$\sigma^{\lambda}=\frac{g_{2 \mathrm{p}}}{g_{1 \mathrm{~s}}} \frac{\lambda_{\mathrm{D}}^{4}}{8 \pi c} A_{2 \mathrm{p} \rightarrow 1 \mathrm{~s}}$

With the known values for the resonance wavelength $\lambda_{\mathrm{D}}=121.5354 \mathrm{~nm}$, the transition probability $A_{2 \mathrm{p} \rightarrow 1 \mathrm{~s}}=6.265 \times 10^{8} \mathrm{~s}^{-1}$ from online databases of the National Institute of Standards and Technology (NIST, http://www.nist.gov) and with $g_{2 \mathrm{p}} / g_{1 \mathrm{~s}}=3$ we find $\sigma^{\lambda}=5.442 \times 10^{-23} \mathrm{~cm}^{3}$.

The fluorescence characteristic $K(\theta)$, here normalized according to $\int K(\theta) \mathrm{d} \Omega=1$, is a function of the angle between the direction of observation and the axis of quantization (the direction of polarization of the exciting radiation in our case)

$K(\theta)=\frac{1}{4 \pi} \frac{3}{3-P_{\perp}}\left(1-P_{\perp} \cos ^{2} \theta\right)$.

For a two-level system and for the case of observation transverse to the direction of polarization the resulting degree of polarization $P_{\perp}$ can be expressed in dependence on the angular momentum quantum numbers $J_{1}$ and $J_{2}$ by Wigner- $6 \mathrm{~J}$ symbols (see $[5,6])$ :

$$
\begin{aligned}
& P_{\perp}=\frac{3 W}{2+W}, \\
& W=3\left(2 J_{2}+1\right)\left\{\begin{array}{ccc}
J_{2} & J_{2} & 2 \\
1 & 1 & J_{1}
\end{array}\right\}^{2} .
\end{aligned}
$$

We obtain after averaging over the fine-structure components according to the statistical weights $4 \pi K\left(\frac{\pi}{2}\right)=\frac{7}{6}$.

\subsection{Calibration with Rayleigh scattered radiation from argon}

The value of the product $T \Delta \Omega L$ has to be known for the determination of the absolute density from the D-Lyman- $\alpha$ fluorescence signal. It is easier and more reliable to compare the fluorescence signal with a Rayleigh scattered signal from a suitable noble gas, detected under otherwise identical conditions, rather than trying to measure these quantities one by one. For such a Rayleigh scattering measurement the number of scattered photons per laser pulse is given by

$\phi_{\mathrm{R}}=T \Delta \Omega n_{\mathrm{R}} L \frac{\mathrm{d} \sigma}{\mathrm{d} \Omega} \phi_{L}$, where $n_{\mathrm{R}}$ stands for the density of the reference gas and $\mathrm{d} \sigma / \mathrm{d} \Omega$ for the differential Rayleigh scattering cross-section [7] given by

$\frac{\mathrm{d} \sigma}{\mathrm{d} \Omega}(\lambda, \theta)=\frac{9 \pi^{2}}{\lambda^{4} n_{\mathrm{R}, 0}^{2}}\left(\frac{\kappa^{2}(\lambda)-1}{\kappa^{2}(\lambda)+2}\right)^{2} \sin ^{2}(\theta)$.

We chose argon for this calibration procedure because its refractive index is known to be $\kappa(121.6 \mathrm{~nm})=1.000502$ at $n_{\mathrm{R}, 0}=2.687 \times 10^{19}$ $\mathrm{cm}^{-3}[8,9]$. The corresponding Rayleigh crosssection for transverse observation amounts to $\mathrm{d} \sigma /$ $\mathrm{d} \Omega=6.265 \times 10^{-25} \mathrm{~cm}^{2}$.

The comparison of the spectrally integrated fluorescence signal (Eq. (1)) with the Rayleigh scattered signal (Eq. (5)) yields the atomic density according to

$n=\frac{\int\left(\phi_{\mathrm{F}} / \phi_{\mathrm{L}}\right) \mathrm{d} \lambda}{K \sigma^{\lambda}} \frac{(\mathrm{d} \sigma / \mathrm{d} \Omega)}{\left(\phi_{\mathrm{R}} / \phi_{\mathrm{L}}\right)} n_{\mathrm{R}}$.

\subsection{Titration with $\mathrm{NO}_{2}$}

The application of the titration procedure requires only a relative measurement of the D-Lyman$\alpha$ fluorescence signal. A variable $\mathrm{NO}_{2} / \mathrm{He}$-flux is added to the main flux so that a fraction of the atomic species is destroyed according to the fast chemical reaction

$\mathrm{H}(\mathrm{D})+\mathrm{NO}_{2} \rightarrow \mathrm{OH}(\mathrm{OD})+\mathrm{NO}$.

If the reaction is complete (in the sense that per reactive molecule one atom is destroyed), a linear decrease of the fluorescence signal with increasing flux of titration gas is expected until the signal amplitude becomes zero at the endpoint of titration. The flux of the reactive molecules $\phi_{\mathrm{T}}$ equals the initial flux of the atomic species at this point. The atomic density can then be found on the basis of the total flux $\phi_{\text {tot }}$, the total pressure $p$ and the gas temperature $T$ according to:

$n_{\mathrm{D}+\mathrm{H}}=\frac{p}{k T} \frac{\phi_{\mathrm{T}}}{\phi_{\mathrm{tot}}}$.

A more detailed consideration of the rate equations

$\frac{\mathrm{d}}{\mathrm{d} t} n_{\mathrm{D}+\mathrm{H}}=\frac{\mathrm{d}}{\mathrm{d} t} n_{\mathrm{NO}_{2}}=-k n_{\mathrm{D}+\mathrm{H}} n_{\mathrm{NO}_{2}}$ 
with the reaction constant $k$ leads to the timedependent solution

$n_{\mathrm{D}+\mathrm{H}}(t)=\frac{n_{\mathrm{D}+\mathrm{H}}(0)}{1+k n_{\mathrm{D}+\mathrm{H}}(0) t}$

for equal starting densities $n_{\mathrm{D}+\mathrm{H}}(0)=n_{\mathrm{NO}_{2}}(0)$. In order to reach a complete termination of the reaction and a well-defined titration endpoint, we have to fulfill the condition $k n_{\mathrm{D}+\mathrm{H}}(0) t \gg 1$. The reaction time $t$ is connected with the reaction length $s$, the cross-section $A$ of the flow tube and the total volume flux $\phi_{\mathrm{V}, \text { tot }}$ according to

$t=\frac{s A}{\phi_{\mathrm{V}, \mathrm{tot}}}$.

This allows, together with the reaction constant $k=1.3 \times 10^{-10} \mathrm{~cm}^{3} \mathrm{~s}^{-1}$ for room temperature [1], to verify the conditions stated on the basis of the result of the titration measurement.

\section{Results}

We have verified the assumption of optical transparency of the D-Lyman- $\alpha$ transition through the medium by measuring the transmitted intensity for standard conditions of the flow tube reactor (He flux $710 \mathrm{sccm}, 0.5 \% \mathrm{D}_{2} / \mathrm{H}_{2}$ flux $10 \mathrm{sccm}$, total pressure $930 \mathrm{~Pa}$ and microwave power $80 \mathrm{~W}$ ). Fluorescence and Rayleigh scattering measurements with variation of focussing of the excitation radiation led to identical results so that saturation can be excluded. Quenching of the excited atoms can also be neglected since the gas flow contains mainly helium which is a very inefficient quenching partner. The lifetime of the excited state is very short, in addition. A rough estimate of the effective quenching rate, based on quenching coefficients for collision of $\mathrm{H}(n=3)$-atoms with $\mathrm{H}_{2}$ and $\mathrm{He}$ [3], results in a value 60 times below the fluorescence rate.

Fig. 3 shows a measured excitation profile of the D-Lyman- $\alpha$ resonance. The resulting profile (solid line) was numerically determined. It is composed of two Gaussian profiles (dashed lines) of equal width with an amplitude ratio of 2:1 and a relative displacement of $\Delta v=0.366 \mathrm{~cm}^{-1}$ of the $2 \mathrm{p}^{2} \mathrm{P}_{J}$ fine-structure components as predicted by

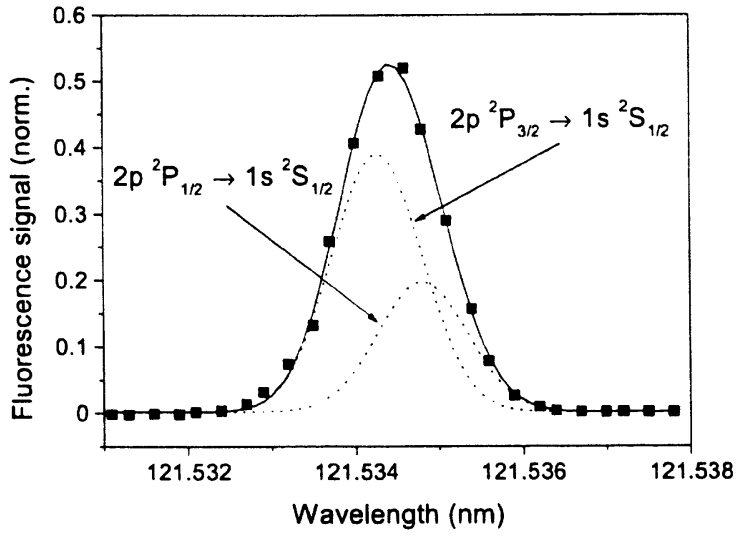

Fig. 3. Measured line profile of the D-Lyman- $\alpha$ transition.

theory. Taking into account a Gaussian line profile of the exciting radiation of width $0.27 \mathrm{~cm}^{-1}$, determined from an absorption profile of the krypton transition $4 \mathrm{p}^{61} \mathrm{~S}_{0} \rightarrow 5 \mathrm{~s}[3 / 2]_{1}$ at $\lambda=123.6 \mathrm{~nm}$ [4], the deconvolution of our measurement gives a translational temperature of $\approx 350 \mathrm{~K}$. This shows that the deuterium atoms are reasonably well thermalized by the cold carrier gas before the point of detection in the flow tube.

Fig. 4 shows the measured Rayleigh scattered signal at the wavelength of the D-Lyman- $\alpha$ resonance as a function of the argon pressure with which the flow tube reactor was filled. This figure comprises three series of measurements taken at different days after exchange of the laser dye mixture. The

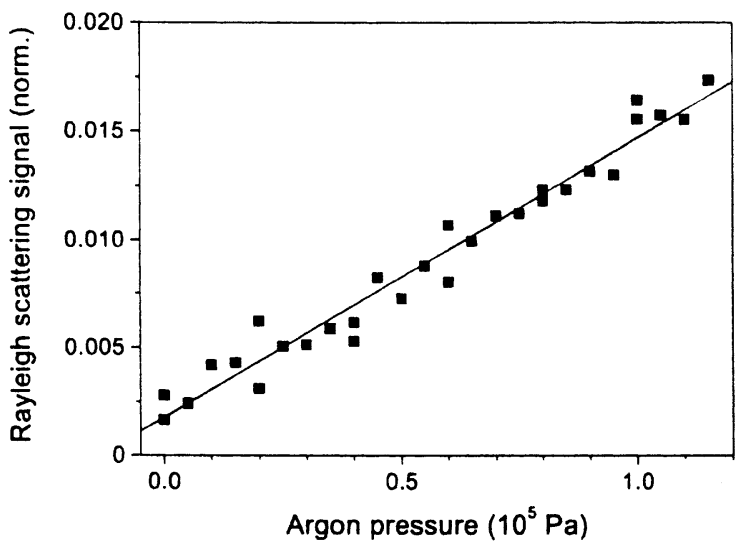

Fig. 4. Rayleigh scattered signal of argon versus pressure at $\lambda=121.535 \mathrm{~nm}$. 
linear dependence is observed even for pressures higher than atmospheric; the reproducibility of the measurements is obvious. The straight line has been adapted numerically; the ordinate intercept is caused by parasitic radiation and has to be subtracted. Comparing the spectrally integrated fluorescence signal with the Rayleigh scattered signal yields an atomic deuterium density of $n_{\mathrm{D}}=$ $1.86 \times 10^{10} \mathrm{~cm}^{-3}$ according to (Eq. (7)) from which a total density of $n_{\mathrm{D}+\mathrm{H}}=3.72 \times 10^{12} \mathrm{~cm}^{-3}$ is inferred on the basis an isotope fraction of $0.5 \%$.

Fig. 5 shows the measured D-Lyman- $\alpha$ fluorescence signal on resonance as a function of the $\mathrm{NO}_{2} / \mathrm{He}$ flux. The titration endpoint is determined by extrapolation to zero signal amplitude as $\phi_{\mathrm{T}}=0.017 \pm 0.002 \mathrm{sccm}$ on the basis of an $\mathrm{NO}_{2}$ fraction of $1.47 \%$. With the gas temperature $T=300 \mathrm{~K}$, the total flux $\phi_{\mathrm{tot}}=710 \mathrm{sccm}$ and the total pressure $p=930 \mathrm{~Pa}$ we obtain from (Eq. (9)) a total atomic density of $n_{\mathrm{D}+\mathrm{H}}=(5.4 \pm 0.6) \times$ $10^{12} \mathrm{~cm}^{-3}$. Using this result one can verify the criterion for completeness of the reaction mentioned above. The product $k n_{\mathrm{D}+\mathrm{H}} t$ assumes the value of $\approx 9$ for a reaction time $t \approx 13 \mathrm{~ms}$. This explains the deviation of the signal from the linear decay in the immediate vicinity of the titration endpoint. Surprisingly, the measured signal exhibits a flat plateau far beyond the titration endpoint before the signal finally reaches zero. This is in contrast to previous

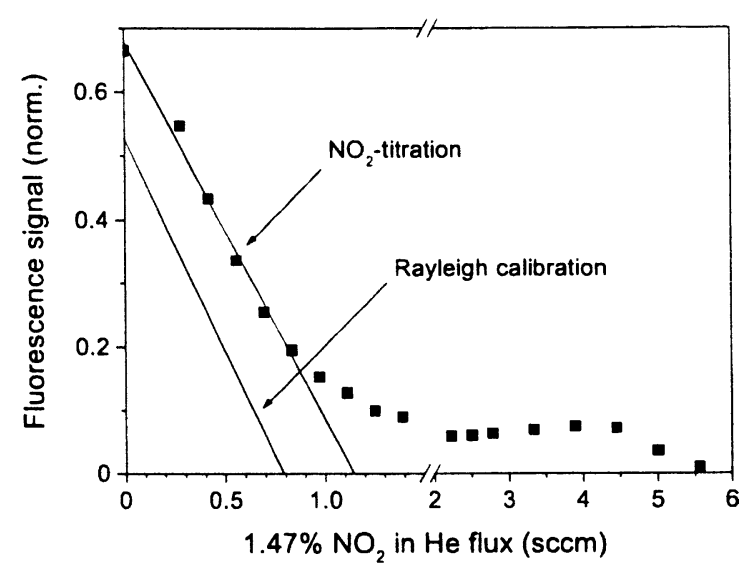

Fig. 5. Measured titration curve for $710 \mathrm{sccm} \mathrm{He}, 10 \mathrm{sccm} 0.5 \%$ $\mathrm{D}_{2} / \mathrm{H}_{2}$ fluxes at $930 \mathrm{~Pa}$ total pressure and $80 \mathrm{~W}$ microwave power in comparison with the results of the Rayleigh calibration. experiments performed in our group [3] where the $\mathrm{H}$ density in hydrogen discharges was investigated with TALIF (two-photon absorption laser-induced fluorescence). An identical flow tube reactor was used for calibration - without deuterium admixture but under otherwise identical conditions including helium as carrier gas in both flows. No plateau was seen in the titration curve in this case. It can, therefore, be excluded that the plateau is due to surface or gas recirculation effects. A possible production of $\mathrm{D} / \mathrm{H}$-atoms by a post-discharge reaction of metastable He with $\mathrm{D}_{2} / \mathrm{H}_{2}$ would lead to an increased but constant atomic flux independent on the flux of the titration gas. Although the appearance of this late plateau phase is of no concern for the applicability of the method, we made an attempt to unravel its origin by modelling the secondary reactions among the species that seemed likely to be involved, namely $\mathrm{NO}_{2}, \mathrm{NO}, \mathrm{H}_{2}, \mathrm{D}_{2}, \mathrm{H}, \mathrm{D}, \mathrm{OH}$, $\mathrm{OD}, \mathrm{H}_{2} \mathrm{O}, \mathrm{D}_{2} \mathrm{O}, \mathrm{HDO}, \mathrm{HD}, \mathrm{O}_{2}, \mathrm{O}$. Although some of the reaction rate coefficients (see NIST online databases) are different for the isotopically substituted species, no plateau occured in the modeled titration curves. Another difference to the common situation of $\mathrm{H}$-atom detection and calibration besides the presence of deuterium is that the photon energy for the VUV-LIF measurement is about twice as large as with TALIF. A direct non-resonant generation of $\mathrm{D}^{*}(n=2)$ by photo-dissociation of $\mathrm{OD}, \mathrm{D}_{2}, \mathrm{HD}$ or HDO can be excluded, because no signal was observed outside the D-Lyman- $\alpha$ resonance. A two step process - e.g., the generation of $\mathrm{D}(n=1)$ by photo-dissociation with subsequent excitation to $n=2$ - is considered to be extremely inefficient. We would like to stress again that the observed plateau is far outside the range of extrapolation and has no influence on the result of the titration measurement.

In addition to the measured titration curve Fig. 5 also represents the corresponding dependence obtained by Rayleigh calibration assuming a $\Delta\left[\mathrm{NO}_{2}\right] / \Delta[\mathrm{H}]$ stoichiometry of 1 for a direct validation of the titration method. The discrepancy between the two slopes is less than $10 \%$ indicating that in this case the titration measurement on the basis of the assumed stoichiometry is reliable within an error that corresponds to the uncertainty of the titration endpoint. Note also that obviously 
in both cases the flow tube reactor was producing different absolute atomic densities.

\section{Conclusions}

Our comparative measurements result in the following statements. The quality of the Rayleigh calibration allows us to measure atomic hydrogen densities by laser-induced fluorescence in the vacuum UV with an accuracy probably better than $10 \%$. Repeated LIF measurements show that even after several hours, during which the external parameters of the flow-tube reactors are kept constant, there are long-term drifts of the atomic hydrogen density on the order of $30 \%$. A characteristic time scale for these drifts to occur is on the order of 30-60 min. The titration process itself exhibits a nonsystematic variation of the extrapolated titration endpoint on the order of $30 \%$. This information applies, if the first 5-6 titration points are taken with time intervals of typically $3 \mathrm{~min}$. The external parameters have reached stationary values at this time. Increasing the time between the measurement of the various titration points may possibly improve the result of the extrapolation; at the risk, however, that one will instead measure a long-term drift.

We conclude that the method of $\mathrm{H}+\mathrm{NO}_{2}$ titration - if carefully carried out - is suitable for absolute calibration of atomic hydrogen densities in the limits stated.

\section{References}

[1] D.W. Setser, Reactive Intermediates in the Gas Phase Generation and Monitoring, Academic, New York, 1979.

[2] A. Goehlich, T. Kawetzki, H.F. Döbele, J. Chem. Phys. 108 (1998) 9362.

[3] K. Niemi, V. Schulz-von der Gathen, H.F. Döbele, J. Phys. D 34 (2001) 2330.

[4] D. Wagner, B. Dikmen, H.F. Döbele, Rev. Sci. Instrum. 67 (1996) 1800.

[5] P.P. Feofilov, The Physical Basis of Polarized Emission, Consultants Bureau, New York, 1961.

[6] I. Sobelman, Atomic Spectra and Radiative Transitions, Springer, Berlin, 1979.

[7] C.M. Penney, J. Opt. Soc. Am. 59 (1969) 34.

[8] G.I. Chashchina, V.I. Gladushchak, E.Y. Shreider, Opt. Spectrosc. 24 (1968) 542.

[9] A. Bideau-Mehu, Y. Guern, R. Abjean, A. Johannin-Gilles, J. Quant. Spectrosc. Radiat. Transfer 25 (1981) 395. 